\title{
Calculation of filtration of polydisperse suspension in a porous medium
}

\author{
Ludmila Kuzmina ${ }^{1}$, and Yuri Osipov, ${ }^{2, *}$ \\ ${ }^{1}$ National Research University Higher School of Economics, 101000 Moscow, Russia \\ ${ }^{2}$ Moscow State University of Civil Engineering, 129337 Moscow, Russia
}

\begin{abstract}
The problem of filtering the suspension in a porous medium is considered. The proposed model for the transport of particles of different sizes is a generalization of deep bed filtration model for a monodisperse suspension with size-exclusion particle capture mechanism. Exact and asymptotic solutions are constructed at the filter inlet and on the concentration front of the suspended and retained particles. Numerical calculation for a suspension with 2-size particles shows that the distribution of deposit in the filter depends on the particle size.
\end{abstract}

\section{Introduction}

Filtration problem is an essential part of the underground hydrodynamics, its study is necessary for the design and construction of dams and hydraulic structures [1]. During filtering of the suspension in the porous medium some particles are retained in the pores and form a deposit. Depending on the physical properties of the porous medium and the suspension particle retention may be caused by diffusion, electrostatic and gravitational forces, etc [2]. If particle and pore size distributions overlap, the predominant particle retention mechanism is size-exclusion [3]. It is assumed that the particles pass freely through the larger pores and get stuck at the inlet of pores which are smaller than the particle diameter.

The classical model of deep bed filtration considers the flow of fluid with 1-size particles in a porous media with constant values of porosity and permeability [4]. In a modified filtration model the porosity and permeability of the filter decrease with increasing deposit. Exact and asymptotic solutions of this problem are obtained in [5-9]. Examples of numerical calculation see in [10]. The filtration models for monodisperse suspension are confirmed by many experimental data [11, 12]. However, there are laboratory studies, the results of which can not be explained within the framework of this model [13].

In this paper one-dimensional filtration of polydisperse suspension in a porous medium is considered. Suspended solid particles of the suspension differ, for example, by their sizes. All types of particles transported by fluid flow move with the same velocity, porosity and permeability of the porous medium depend on the particles deposits. It is assumed that filter coefficients depend on the type of particles. Filtering of each type of particles is

\footnotetext{
${ }^{*}$ Corresponding author: yuri-osipov@mail.ru
} 
determined by mass balance equation and kinetic equation of particle capture, similar to the equations for a monodisperse suspension. These equations form a system of $2 n$ quasi-linear differential equations of the first order. It is assumed that the suspension with constant concentrations of suspended particles is supplied at the filter inlet, and at the initial time the porous medium does not contain any particles.

\section{Governing equations}

In the domain $\Omega=\{0<x<1, t>0\}$ the suspended and retained particles concentrations $C_{i}(x, t), S_{i}(x, t), i=1, \ldots, n$ satisfy the equations

$$
\begin{gathered}
\frac{\partial}{\partial t}\left(g(\vec{S}) C_{i}\right)+\frac{\partial}{\partial x}\left(f(\vec{S}) C_{i}\right)+\frac{\partial S_{i}}{\partial t}=0 \\
\frac{\partial S_{i}}{\partial t}=\Lambda_{i}(\vec{S}) C_{i} ; i=1, \ldots, n
\end{gathered}
$$

with border and initial conditions

$$
\begin{aligned}
& x=0: C_{i}=p_{i} \\
& t=0: C_{i}=0, S_{i}=0
\end{aligned}
$$

Here $\vec{S}=\left(S_{1}, \ldots, S_{n}\right)$; porosity $g(\vec{S})$, permeability $f(\vec{S})$, and the filter coefficients $\Lambda_{i}(\vec{S})$ are continuous positive functions; $p_{i}>0$ are constant.

Concentration front of suspended and retained particles moves with constant velocity

$$
v=f(\overrightarrow{0}) / g(\overrightarrow{0})
$$

and divides the domain $\Omega$ into two subdomains $\Omega_{0}=\{0<x<1,0<t<x / v\}$ and $\Omega_{1}=\{0<x<1, t>x / v\}$. In $\Omega_{0}$ the system (1) - (4) has a zero solution; in $\Omega_{1}$ the concentrations $C_{i}, S_{i}$ are positive. Since the conditions (3) and (4) disagree at the origin, the solution $C_{i}(x, t)$ has a strong discontinuity on the characteristic line $t=x / v$ - the concentration front. The solution $S_{i}(x, t)$ is continuous in $\Omega$ and has a weak break on the concentration front.

Consider the condition on the concentration front

$$
\left.S_{i}\right|_{t=x / v}=0 \text {. }
$$

In the domain $\Omega_{1}$ the problem (1) - (4) is equivalent to the Goursat problem (1) - (3), (6).

\section{Exact and asymptotic solution on the concentration front}

Differentiating the terms of equations (1)

$$
g(\vec{S}) \frac{\partial C_{i}}{\partial t}+C_{i} \sum_{k=1}^{n} \frac{\partial g}{\partial S_{k}} \cdot \frac{\partial S_{k}}{\partial t}+f(\vec{S}) \frac{\partial C_{i}}{\partial x}+C_{i} \sum_{k=1}^{n} \frac{\partial f}{\partial S_{k}} \cdot \frac{\partial S_{k}}{\partial x}+\frac{\partial S_{i}}{\partial t}=0 ; \quad i=1, \ldots, n
$$


Denote

$$
f_{0}=f(\overrightarrow{0}), g_{0}=g(\overrightarrow{0}), \lambda_{i}=\Lambda_{i}(\overrightarrow{0}), g_{i}^{\prime}=\frac{\partial g}{\partial S_{i}}(\overrightarrow{0}), f_{i}^{\prime}=\frac{\partial f}{\partial S_{i}}(\overrightarrow{0})
$$

According to (2), (6) on the concentration front the equations (7) take the form

$$
g_{0} \frac{\partial C_{i}}{\partial t}+C_{i} \sum_{k=1}^{n} g_{k}^{\prime} \lambda_{k} C_{k}+f_{0} \frac{\partial C_{i}}{\partial x}+C_{i} \sum_{k=1}^{n} f_{k}^{\prime} \frac{\partial S_{k}}{\partial x}+\lambda_{i} C_{i}=0 ; i=1, \ldots, n
$$

The transition to the characteristic variables

$$
\tau=t-\frac{g_{0}}{f_{0}} x, y=x
$$

gives

$$
\frac{\partial C_{i}}{\partial t}=\frac{\partial C_{i}}{\partial \tau} ; \frac{\partial C_{i}}{\partial x}=\frac{\partial C_{i}}{\partial y}-\frac{g_{0}}{f_{0}} \frac{\partial C_{i}}{\partial \tau}
$$

Differentiating (6) along the concentration front, we obtain

$$
\left.\frac{\partial S_{k}}{\partial x}\right|_{t=x / v}=-\frac{g_{0}}{f_{0}} \lambda_{k} C_{k}, k=1, \ldots, n .
$$

Substitution of (10), (11) in (8) gives a system of equations

$$
f_{0} \frac{\partial C_{i}}{\partial y}+\lambda_{i} C_{i}+C_{i} \sum_{k=1}^{n} A_{k} \lambda_{k} C_{k}=0 ; A_{k}=g_{k}^{\prime}-\frac{f_{k}^{\prime}}{v}, i=1, \ldots, n
$$

with conditions

$$
y=0: C_{i}=p_{i}
$$

The solution of Bernoulli's equations (12), (13)

$$
C_{i}(y)=\frac{p_{i} e^{-\frac{\lambda_{i}}{f_{0}} y}}{1+\sum_{k=1}^{n} A_{k} p_{k}\left(1-e^{-\frac{\lambda_{k} y}{f_{0}}}\right)}, i=1, \ldots, n .
$$

(14) presents formulas for the suspended particles concentrations on the concentration front.

From (2)

$$
\left.\frac{\partial S_{i}}{\partial \tau}\right|_{\tau=0}=\lambda_{i} C_{i}(y) ; i=1, \ldots, n .
$$

Therefore, the main terms of the asymptotics near the concentration front have the form

$$
C_{i}(y, \tau)=C_{i}(y)+O(\tau) ; \quad S_{i}(y, \tau)=\lambda_{i} C_{i}(y) \tau+O\left(\tau^{2}\right)
$$




\section{Example. Model of 2-size particle filtration}

Consider a filtration model of the suspension with 2-size particles in the porous medium [14]. The coefficients of the equations (1), (2)

$$
\begin{gathered}
g(\vec{S})=a-S_{1}-S_{2}, \quad a>0 ; \quad f(\vec{S})=b\left(a-S_{1}-S_{2}\right)^{3}, \quad b>0 ; \\
\Lambda_{i}(\vec{S})=\lambda_{i}-S_{1}-S_{2}, \quad \lambda_{i}>0 ; \quad i=1,2 .
\end{gathered}
$$

Then the velocity of concentration front propagation is $v=a^{2} b$; coefficients $A_{k}=2, k=1,2$.

Summing equations (2) and substituting (3) and (18) we obtain an equation for the total deposit $S=S_{1}+S_{2}$ at the filter inlet $x=0$

$$
\frac{\partial S}{\partial t}=\lambda_{1} p_{1}+\lambda_{2} p_{2}-\left(p_{1}+p_{2}\right) S ;\left.S\right|_{t=0}=0
$$

The solution of (19)

$$
S(0, t)=\frac{\lambda_{1} p_{1}+\lambda_{2} p_{2}}{p_{1}+p_{2}}\left(1-e^{-\left(p_{1}+p_{2}\right) t}\right)
$$

According to (16) the asymptotic solution near the concentration front in the variables $(x, t)$

$$
\begin{gathered}
C_{i}(x, t)=\frac{p_{i} e^{-\frac{\lambda_{i}}{a^{3} b}}}{1+2 \sum_{k=1}^{2} p_{k}\left(1-e^{-\frac{\lambda_{k}}{a^{3} b} x}\right)}+O\left(t-\frac{x}{v}\right) ; \\
S_{i}(x, t)=\frac{\lambda_{i} p_{i} e^{-\frac{\lambda_{i}}{a^{3} b}}}{1+2 \sum_{k=1}^{2} p_{k}\left(1-e^{-\frac{\lambda_{k}}{a^{3} b} x}\right)}\left(t-\frac{x}{v}\right)+O\left(t-\frac{x}{v}\right)^{2}, i=1,2 .
\end{gathered}
$$

Calculations of retained particles concentrations were made for

$$
\lambda_{1}=1, \lambda_{2}=300, p_{1}=0.25, p_{2}=0.002, a=4, b=1, \tau=t-\frac{x}{v}=1 .
$$

The graph of total deposit $S(0, t)$ at the filter inlet is shown in figure 1 .

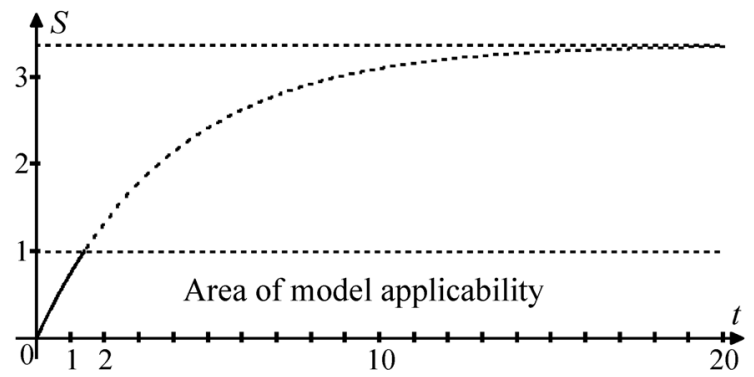

Fig. 1. Total deposit at the filter inlet. 
Figure 2 shows the graphs of retained particles concentrations $S_{1}, S_{2}$ and total retained concentration $S=S_{1}+S_{2}$.

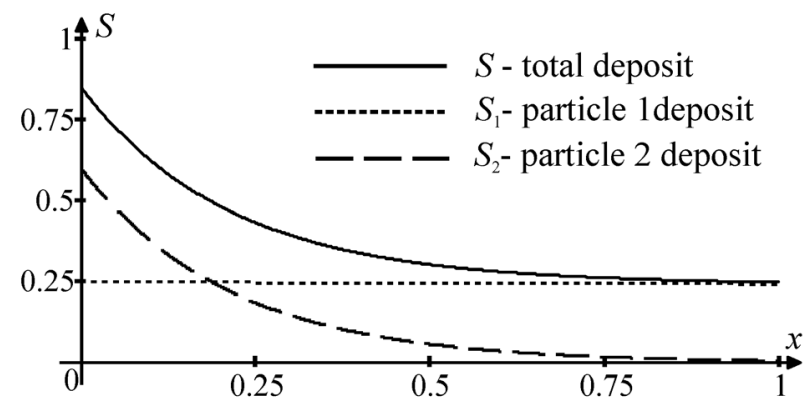

Fig. 2. Retained concentrations near the concentration front.

\section{Conclusion}

The exact and asymptotic solutions of the filtration problem for polydisperse suspension in a porous medium are constructed on the concentration front. The multiparticle model of size-exclusion particle retention allows differing particle deposition rate for the particles of different sizes. For suspension with 2-size solid particles computation of retained particles concentration near the concentration front was carried out. Physical conditions $\Lambda_{i}(\vec{S})>0 ; i=1,2$ limit the applicability of the example in section 4 (see Figure 1). Figure 2 shows that the penetration depth of retained particles in a porous medium decreases with increasing particle size. Large particles are stuck close to the filter inlet and the smaller particles are uniformly deposited throughout the porous medium.

It is of interest to construct next terms of asymptotic solution and to compare it with laboratory data. This will be considered elsewhere.

\section{References}

1 K.S. Basniev, N.M. Dmitriev, Mechanics of fluid flow (Wiley, 2012)

2 C. Tien, B.V. Ramarao, Granular filtration of aerosols and hydrosols (Elsevier, Amsterdam, 2007)

3 A. Badalyan, Z. You, K. Aji, P. Bedrikovetsky, T. Carageorgos, A. Zeinijahromi, Rev. Sci. Instrum 85, ID 015111 (2014).

4 J. P. Herzig, D. M. Leclerc, and P. Legoff, Ind. Eng. Chem. 62 (1970)

5 Z. You, P. Bedrikovetsky, L. Kuzmina, Abstr. Appl. Anal. 2013, ID 680693 (2013)

6 Z. You, Y. Osipov, P. Bedrikovetsky, L. Kuzmina, Chem. Eng. J. 258 (2014)

7 L.I. Kuzmina, Yu.V. Osipov, Procedia Eng. 111 (2015)

8 L.I. Kuzmina, Yu.V. Osipov, Procedia Eng. 153 (2016)

9 L.I. Kuzmina, Yu.V. Osipov, Int. J. Comp. Civil Str. Eng. 12, 1 (2016)

10 Yu.P. Galaguz, G.L. Safina, Procedia Eng. 153 (2016)

11 P.G. Bedrikovetsky, D. Marchesin, F. Checaira, A.L. Serra, E. Resende, Petrol. Sci. Eng. 32, 3 (2001)

12 D. Mays, J.R. Hunt, J. Environ. Sci. Technol. 39 (2005)

13 L. Bailey, E. Boek, S. Jacques, SPE 54762, (1999)

14 V.I. Golubev, D.N. Michailov, Trudi MFTI 3, 2 (2011) 